\title{
Spontaneously remitting insulin autoimmune syndrome in a patient taking alpha-lipoic acid
}

\author{
D Cappellani', C Sardella', M C Campopiano1, A Falorni², P Marchetti and E Macchia1 \\ 1Unit of Endocrinology, Department of Clinical and Experimental Medicine, University of Pisa, Pisa, Italy, ${ }^{2 S e c t i o n}$ of \\ Internal Medicine and Endocrine and Metabolic Sciences, Department of Medicine, University of Perugia, Perugia, \\ Italy, and ${ }^{3}$ Division of Metabolism and Cell Transplantation, Department of Clinical and Experimental Medicine, \\ University of Pisa, Pisa, Italy
}

Correspondence

should be addressed

to D Cappellani

Email

d.cappellani@hotmail.it

\section{Summary}

Insulin autoimmune syndrome (IAS), or Hirata disease, is a rare hypoglycaemic disorder caused by the presence of high titer of insulin autoantibodies (IAA) in patients without previous exposure to exogenous insulin. Even though its pathogenesis is not fully understood, striking evidences link IAS to previous exposure to sulphydryl-containing medications, like alpha-lipoic acid, a widely used nutritional supplement. Although challenging, a careful differential diagnosis from other causes of hyperinsulinaemic hypoglycaemia (such as insulinoma) is mandatory, since these conditions require different therapeutic approaches. In the present study, we report a 35-year-old woman originally from Sri Lanka who was referred to our University Hospital on suspicion of occult insulinoma. Her medical history was positive for endometriosis, treated with estroprogestins and alpha-lipoic acid. The latter supplement was begun 2 weeks before the first hypoglycaemic episode. Our tests confirmed the presence of hypoglycaemia associated with high insulin and C-peptide concentrations. When insulin concentrations were compared using different assays, the results were significantly different. Moreover, insulin values significantly decreased after precipitation with polyethylene glycol. An assay for IAA proved positive $(530 \mathrm{U} / \mathrm{mL})$. A genetic analysis revealed the presence of HLA-DRB $1 * 04,15$, an immunogenetic determinant associated with IAS. On the basis of clinical data we avoided a first-line approach with immunosuppressive treatments, and the patient was advised to modify her diet, with the introduction of frequent low-caloric meals. During follow-up evaluations, glucose levels (registered trough a flash glucose monitoring system) resulted progressively more stable. IAA titer progressively decreased, being undetectable by the fifteenth month, thus indicating the remission of the IAS.

\section{Learning points:}

- Insulin autoimmune syndrome (IAS) is a rare cause of hyperinsulinaemic hypoglycaemia, whose prevalence is higher in East Asian populations due to the higher prevalence of specific immunogenetic determinants. Nevertheless, an increasing number of IAS cases is being reported worldwide, due to the wide diffusion of medications such as alpha-lipoic acid.

- Differential diagnosis of IAS from other causes of hyperinsulinemic hypoglycaemia is challenging. Even though many tests can be suggestive of IAS, the gold standard remains the detection of IAAs, despite that dedicated commercial kits are not widely available.

- The therapeutic approach to IAS is problematic. As a matter of fact IAS is often a self-remitting disease, but sometimes needs aggressive immunosuppression. The benefits and risks of any therapeutic choice should be carefully weighted and tailored on the single patient. 


\section{Background}

Insulin autoimmune syndrome (IAS) is a rare disease, firstly described by Hirata and colleagues in 1970 (1) and characterised by spontaneous hypoglycaemic episodes in the presence of high titer of insulin autoantibodies (IAAs) in patients without previous exposure to exogenous insulin. Hypoglycaemic episodes can occur during fast or after a meal and can be life-threatening.

Mostly due to immunogenetic determinants (2), this condition is the third leading cause of hypoglycaemia in Japan, but case reports or case series are increasingly being published outside this country and in non-Asian populations. Striking evidences link this syndrome to previous exposure to some medications (3), including methimazole, glutathione or alpha-lipoic acid. Like insulin itself, all these drugs are sulphydryl compounds; as a consequence, a molecular mimicry mechanism can be advocated as pathogenetic.

Differential diagnosis from insulinoma is sometimes challenging, but it is important in order to avoid invasive procedures and inappropriate abdominal surgery. The management of IAS is challenging too: on the one side, a high percentage of spontaneous remission is described (estimated in $60-70 \%$ of the untreated patients) (4), on the other side, the potential life-threatening nature of the disease warrants pharmacologic immunosuppressive approaches that are not free from side effects.

\section{Case presentation}

A 35-year-old woman originally from Sri Lanka presented to the Emergency Department of a peripheral primary care hospital for the sudden occurrence of neurological symptoms (asthenia, blurred vision, aphasia, loss of coordination and partial amnesia). Given the finding of a blood glucose of $38 \mathrm{mg} / \mathrm{dL}$, the patient was treated with intravenous glucose administration with prompt resolution of symptoms. For the recurrence of hypoglycaemia during the short-stay observation period, the patient was admitted to the local General Medicine Unit.

A blood test performed during a subsequent hypoglycaemic episode revealed an insulin value higher than $1000 \mu \mathrm{U} / \mathrm{mL}$ with a C-peptide of $31.8 \mathrm{ng} /$ mL. Imaging studies (including abdominal echography, abdominal CT, abdominal RM, 68Ga-DOTATOC PET) did not reveal any lesion suggestive for insulin-secreting tumours. In the belief of an occult insulinoma, the patient was then transferred to our University Hospital for further evaluation and possible surgical management.

\section{Investigation}

The clinical history revealed no previous significant illnesses, except for endometriosis treated with estroprogestins: the patient had recently changed the route of administration of such medications (previously oral route, currently vaginal route) and had begun a cycle of alpha-lipoic acid just 2 weeks before the first hypoglycaemic episode. Physical examination resulted unremarkable: patient height was $1.65 \mathrm{~m}$, weight was $66 \mathrm{~kg}$ and the resulting BMI was $24.2 \mathrm{~kg} / \mathrm{m}^{2}$.

At baseline, routine tests of blood glucose showed $129 \mathrm{mg} / \mathrm{dL}$ and blood insulin $2465 \mu \mathrm{U} / \mathrm{mL}$; the latter result was obtained using an immunochemiluminometric assayICMA (Access Ultrasensitive Insulin, Beckman Coulter Diagnostics). Hormonal examinations were normal and the autoimmune panel was negative.

During another spontaneous hypoglycaemia (blood glucose: $41 \mathrm{mg} / \mathrm{dL}$ ), hyperinsulinemia (blood insulin: $2294 \mu \mathrm{U} / \mathrm{mL}$, ICMA) was found associated with an elevated C-peptide level (C-peptide: $12.36 \mathrm{ng} / \mathrm{mL}$ ).

Insulin concentrations were tested using a different method: with a blood glucose of $62 \mathrm{mg} / \mathrm{dL}$ insulin was $247.8 \mu \mathrm{U} / \mathrm{mL}$ when measured with an ICMA and 37.47 when measured with an immunoradiometric assay (IRMA; Insulin IRMA Kit, Beckman Coulter Diagnostics). As previously described (5) a polyethylene glycol (PEG) precipitation test was performed on various insulin samples, revealing a ten-fold reduction in insulin values (e.g. from 2465 to $256 \mu \mathrm{U} / \mathrm{mL}$ and from 2576 to $128.87 \mu \mathrm{U} / \mathrm{mL}$ respectively).

As we suspected an IAS, a blood sample for diagnosis of IAA was drawn. The assay of these autoantibodies gave a frankly positive result $(530 \mathrm{U} / \mathrm{mL}$; normal values $<0.4$ ) (Fig. 1). At the same time, a genetic analysis was performed, which revealed the presence of HLADRB1*04,15, an immunogenetic determinant already described as associated with IAS (2).

\section{Treatment}

The patient's diet was modified with the introduction of high frequent and low-caloric meals, rich in simple sugars, which was associated to reduced hypoglycaemic episodes. Furthermore, a flash glucose monitoring (FGM) system FreeStyle Libre (Abbott) was inserted, demonstrating a progressively more stable glucose level.

Taking into account the high percentage of spontaneous remission of this syndrome, the absence of other associated autoimmune diseases, the effectiveness 


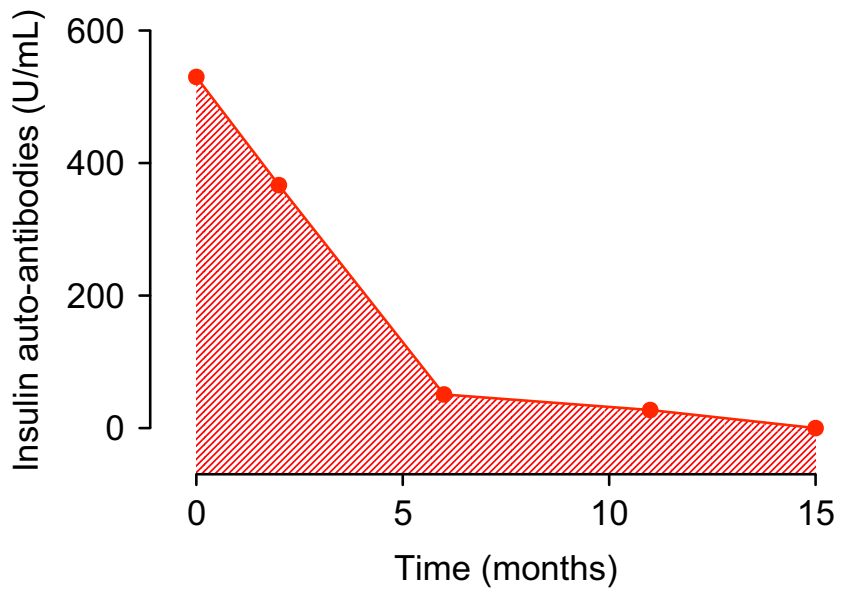

Figure 1

Insulin autoantibodies (IAA) concentrations during the follow-up. On the $X$ axis time is represented in months from the first report of IAA.

of the diet modification according to the FGM and the wish of the patient, we decided not to actively treat the condition, avoiding at least initially immunosuppressive treatments. The patient was dismissed with the indication to continue FGM while following the same diet and repeating blood samples for IAA.

\section{Outcome and follow-up}

The patient came back at months 2, 6, 11 and 15 during the follow-up. Glycaemic levels detected using FGM were proved durably in the acceptable range, without significant hypoglycaemic episodes (Fig. 2). At 6 months from the diagnosis, IAA titer resulted $51 \mathrm{U} / \mathrm{mL}$, with a reduction of $90.4 \%$ from the baseline (Fig. 1).

The patient was then advised to gradually give up the prescribed diet, going back to the pre-admission eating habits. IAA titer gradually decreased, and by the fifteenth month were completely undetectable, indicating the complete remission of the disease (Fig. 1).

\section{Discussion}

This case report provides insight into important issues in diagnosis and management of IAS. IAS is a form of
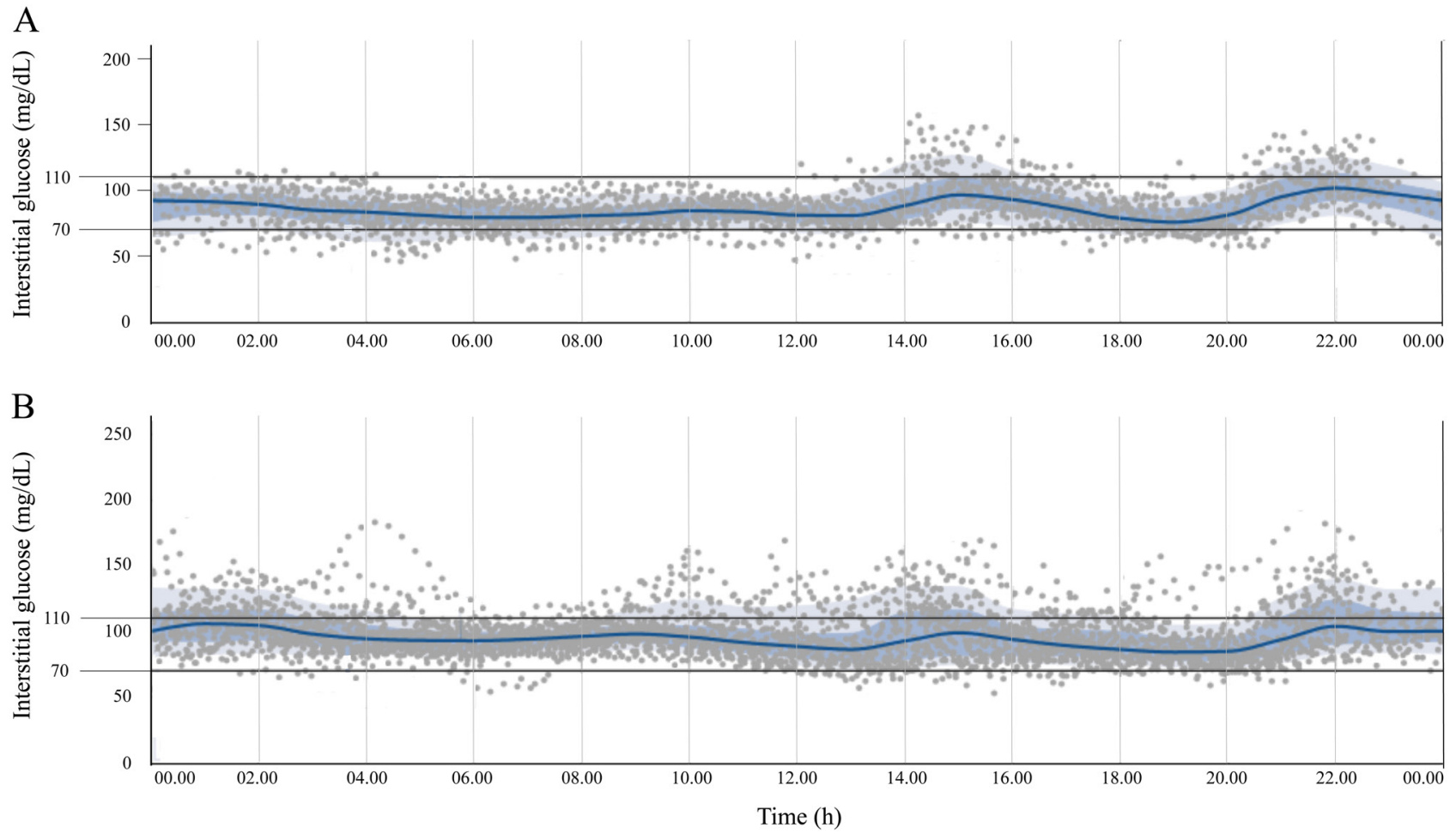

Figure 2

Interstitial glucose concentrations measured with flash glucose monitoring (FGM) system. Panel A: interstitial glucose concentrations during the first month after discharge. Panel B: interstitial glucose concentrations in the sixth month after discharge. Both graphs are modified from the original graphic reports obtained from the FGM system itself. In both panels the grey spots represent single glucose measurements; blue lines represent the mean interstitial glucose concentration; blue-shaded areas represent the interquartile range (25th to 75th percentile); pale-grey areas represent the range from the 10 th to the 90 th percentile. 
hyperinsulinemic hypoglycaemia and is characterised by autoantibodies to endogenous insulin in individuals without previous exposure to exogenous insulin. Since the first description in 1970 (1), more than 380 case of IAS have been reported worldwide (3), mainly in the Japanese population (>90\%) where this disease is nowadays considered the third most common cause of hypoglycaemia (6). The etiopathogenesis of this condition is still a matter of debate. The most widely accepted theory is that IAS results from the interaction of genetic predisposition with environmental triggers, thus leading to the production of IAAs which have a pathogenic role (6). The presence of HLA-DR4 is a wellknown genetic predisposing factor $(2,7)$. IAS is often associated with other autoimmune diseases, so that a genetic predisposition on a larger scale can be inferred. Infections and exposure to certain drugs $(3,5,8)$ have been advocated as possible triggers. All the medications associated with IAS are sulphydril and reducing compounds, thus making conceivable the presence of a molecular mimicry mechanism. The association with the use of alpha-lipoic acid was firstly described in Japan in 2003 and successively extensively reported worldwide $(3,5)$. Hypoglycaemia is probably the consequence of a mismatch between glucose and free insulin concentration, secondary to the binding and release of secreted insulin by IAA $(6,9)$. Clinical manifestations of IAS vary widely in terms of severity, duration of hypoglycaemic attacks and remission rates.

Once hypoglycaemia is confirmed by the presence of the Whipple triad (symptoms or signs of hypoglycaemia associated with low plasma glucose concentration and resolution of those symptoms or signs after the plasma glucose concentration is raised), the differential diagnosis with other hypoglycaemic disorders is challenging (10). In IAS, hypoglycaemia is associated with markedly high insulin (often above $>1000 \mathrm{pmol} / \mathrm{L}$, a value that is rarely seen in insulinomas (9)) and C-peptide concentrations, thus confirming the presence of endogenous hyperinsulinism. The gold standard for the diagnosis of IAS is the detection of IAA, but commercial kits for the detection of IAA are not widely available. As a consequence, alternative diagnostic tools were proposed, such as precipitation with PEG, followed by insulin assay in the supernatant (5).

In our patient, a series of different diagnostic tools were used for the diagnosis of IAS. The baseline biochemical features were suggestive for endogenous hyperinsulinemic hyperglycemia. Contemporarily, the difference in insulin levels registered with different assays were suggestive for a laboratory interference and/or for the presence of IAA complexes, as successively demonstrated by precipitation with PEG. Due to the high suspicion of the presence of IAS, we performed an assay for IAA, and their presence supported the diagnosis. During the follow-up, IAAs were assayed again, revealing a progressive lowering in autoantibody titer. This result was in line with the clinical features reported by the patient and with the glucose profiles registered by the FGM system.

Despite the description of frequent spontaneous remission (8), IAS sometimes needs immunosuppressive treatments. A rational first-line approach consists in small frequent meals with low carbohydrate content in order to reduce postprandial hyperglycaemia and the consequent stimulus to insulin secretion $(9,11)$. According to the published guidelines, the medical treatment of autoimmune hypoglycaemia is problematic (10). Glucocorticoids can be the first medication of choice, where necessary $(4,5)$. Among the other second-line therapeutic options, immunosuppressants (azathioprine and 6-mercaptopurine), monoclonal antibodies (rituximab) (12), plasmapheresis and strategies to reduce insulin release (somatostatin analogues, diazoxide and pancreatectomy), all demonstrated varying efficacy in the management of IAS $(4,11)$. Independently from the kind of therapeutic approach adopted, frequent glucose monitoring during the follow-up seems advisable. Because the application of continuous glucose monitoring to this rare condition has been reported as successful (12), we decided to use a FGM in this case. The pros of the FGM is that this system provides a low-cost non-invasive tool for frequent monitoring of glucose concentrations, enhancing the patient self-monitoring. The cons of this system is that it measures glucose concetrations in the interstitium, thus revealing the presence of hypoglycaemia in delay when compared to glucose assays on capillary blood, underestimates hypoglycaemic episodes and does not provide an acoustic alarm for hypoglycaemic episodes.

In conclusion, IAS is a rare condition, defined by the presence of IAA which are the mediators of hyperinsulinemic hypoglycaemia. IAS is interpreted as an autoimmune disease, even though its pathophysiology is still not fully understood. Specifically, the mechanisms underneath the development of IAA are not completely clear and the genesis of hypoglycaemia has not been explained in detail yet. Moreover, it is still unclear why an autoimmune disease undergoes a spontaneous resolution in the majority of cases even though the antigen (insulin itself) is still present. We wonder whether this condition 
could be considered an immune-mediated disease rather than an autoimmune disease.

\section{Declaration of interest}

The authors declare that there is no conflict of interest that could be perceived as prejudicing the impartiality of the research reported.

\section{Funding}

This research did not receive any specific grant from any funding agency in the public, commercial or not-for-profit sector

\section{Patient consent}

Written informed consent for publication of clinical data was obtained from the patient.

\section{Author contribution statement}

D Cappellani, C Sardella, M C Campopiano, P Marchetti and E Macchia were involved in the direct care of the patient. A Falorni was responsible for the IAA assays. D Cappellani and M C Campopiano revised the clinical charts. D Cappellani wrote the initial draft. C Sardella, A Falorni, P Marchetti, E Macchia edited the manuscript. A Falorni, P Marchetti and E Macchia reviewed and approved the final version of the manuscript.

\section{References}

1 Hirata Y, Ishizu H, Ouchi N, Motomura S, Abe M \& Hara Y. Insulin autoimmunity in a case of spontaneous hypoglycemia. Journal of the Japan Diabetes Society 197013 312-320.

2 Uchigata Y, Tokunaga K, Nepom G, Bannai M, Kuwata S, Dozio N, Benson EA, Ronningen KS, Spinas GA, Tadokoro K, et al. Differential immunogenetic determinants of polyclonal insulin autoimmune syndrome (Hirata's disease) and monoclonal insulin autoimmune syndrome. Diabetes 199544 1227-1232. (https://doi.org/10.2337/ diab.44.10.1227)

3 Uchigata Y, Hirata Y \& Iwamoto Y. Drug-induced insulin autoimmune syndrome. Diabetes Research and Clinical Practice 2009 83 e19-e20. (https://doi.org/10.1016/j.diabres.2008.10.015)

4 Uchigata Y, Eguchi Y, Takayama-Hasumi S \& Omori Y. Insulin autoimmune syndrome (Hirata disease): clinical features and epidemiology in Japan. Diabetes Research and Clinical Practice 199422 89-94. (https://doi.org/10.1016/0168-8227(94)90040-X)

5 Gullo D, Evans JL, Sortino G, Goldfine ID \& Vigneri R. Insulin autoimmune syndrome (Hirata disease) in European Caucasians taking alpha-lipoic acid. Clinical Endocrinology 201481 204-209. (https://doi.org/10.1111/cen.12334)

6 Ismail AA. The insulin autoimmune syndrome (IAS) as a cause of hypoglycaemia: an update on the pathophysiology, biochemical investigations and diagnosis. Clinical Chemistry and Laboratory Medicine 201654 1715-1724. (https://doi.org/10.1515/cclm-20151255)

7 Uchigata Y, Omori Y, Nieda M, Kuwata S, Tokunaga K \& Juji T. HLA-DR4 genotype and insulin-processing in insulin autoimmune syndrome. Lancet 1992340 1467. (https://doi.org/10.1016/01406736(92)92654-X)

8 Hirata Y \& Uchigata Y. Insulin autoimmune syndrome in Japan. Diabetes Research and Clinical Practice 199424 (Supplement) S153-S157. (https://doi.org/10.1016/0168-8227(94)90243-7)

9 Redmon JB \& Nuttall FQ. Autoimmune hypoglycemia. Endocrinology and Metabolism Clinics of North America. 199928 603-618, vii. (https://doi.org/10.1016/S0889-8529(05)70090-6)

10 Cryer PE, Axelrod L, Grossman AB, Heller SR, Montori VM, Seaquist ER \& Service FJ. Evaluation and management of adult hypoglycemic disorders: an Endocrine Society Clinical Practice Guideline. Journal of Clinical Endocrinology and Metabolism 200994 709-728. (https://doi.org/10.1210/jc.2008-1410)

11 Wong SL, Priestman A \& Holmes DT. Recurrent hypoglycemia from insulin autoimmune syndrome. Journal of General Internal Medicine 201429 250-254. (https://doi.org/10.1007/s11606-013-2588-9)

12 Saxon DR, McDermott MT \& Michels AW. Novel management of insulin autoimmune syndrome with rituximab and continuous glucose monitoring. Journal of Clinical Endocrinology and Metabolism 2016101 1931-1934. (https://doi.org/10.1210/jc.2016-1097)

Received in final form 25 October 2018 Accepted 22 November 2018 